\title{
HIGH-ACCURACY EQUATION OF EQUINOXES AND VLBI
} ASTROMETRIC MODELLING

\author{
A.M. GONTIER \\ Observatoire de Paris - Central Bureau of I.E.R.S. \\ 61, avenue de l'Observatoire, F-75014 Paris, France. \\ N. CAPITAINE \\ Observatoire de Paris - URA 1125 / CNRS \\ 61, avenue de l'Observatoire, F-75014 Paris, France.
}

ABSTRACT In the course of implementing the use of nonrotating origin (Guinot 1979) in astrometric VLBI, for the transformation between celestial and terrestrial frames, we have compared the new approach with the classical one. We have shown that a difference exists between the two procedures at a few milliarcsecond level; this difference is due to the terms generally neglected when considering the equation of equinoxes in the classical representation of the Earth angle of rotation.

\section{INTRODUCTION}

The analysis of an Earth based observation of a celestial object requires an astrometric model to express the transformation from a geocentric terrestrial frame to a geocentric celestial one. The classical transformation refers to the equinox of date. In a new astronomical modelling approach, we refer to the "nonrotating origin" on the equator of date, proposed by Guinot (1979) and developped by Capitaine (1990). The comparison, in astrometric VLBI, of the two procedures discloses discrepancy due to the terms generally neglected, when considering the equation of equinoxes in the classical procedure.

\section{THE CLASSICAL PROCEDURE FOR THE CONSIDERATION OE THE EARTH ROTATION}

In order to determine the orientation of the Earth for VLBI data analysis, the angle $\mathrm{H}$ of the Earth orientation in space has to be evaluated. In the classical procedure $\mathbf{H}$ (apparent sidereal time) is the hour angle of the direction of the true equinox (i.e. ascending node of the ecliptic on the equator) relative to the prime meridian. The direction of the equinox has periodic perturbations as a consequence of the celestial motion of the Earth pole (precession and nutation). The equation of equinoxes, $\alpha_{E}$, permits the regularization of the sidereal time through the following expression (Woolard 1953):

$$
\mathrm{H}=\mathrm{GMST}+\alpha_{\mathrm{E}}
$$

where GMST is the Greenwich mean sidereal time . 
In the usual procedure, only the first term in the development of $\alpha_{E}$ is considered: $\Delta \psi \cos (\varepsilon)$, where $\Delta \psi$ is the nutation in ecliptic longitude and $\varepsilon$ is the mean obliquity of the ecliptic.

\section{THE COMPLETE PROCEDURE}

In the new procedure based on the nonrotating origin, the Earth angle of rotation is represented by the "stellar angle" $\theta$, which is the angle between the direction of the nonrotating origin defined on the instantaneous equator (Guinot 1979) and the prime meridian. In opposition to the hour angle of the equinox used in the classical procedure, the stellar angle is not sensitive to precession and nutation (Capitaine $e t$ al. 1986).

In order to compare this procedure with the classical one, it is necessary to use the complete expression of the equation of equinoxes as given by Woolard (1953) :

$$
\alpha_{\mathrm{E}}=\Delta \psi \cos (\varepsilon)-\dot{\psi} \sin (\varepsilon) \int_{\mathrm{t}_{0}}^{\mathrm{t}} \Delta \varepsilon \mathrm{d}-\sin (\varepsilon) \int_{\mathrm{t}_{0}}^{\mathrm{t}} \frac{\mathrm{d} \Delta \psi}{\mathrm{d}} \mathrm{d}
$$

for which Aoki and Kinoshita (1983) have developped a numerical expression, which is at the level of $5.10^{-5 "}$ :

$$
\alpha_{E}=\Delta \psi \cos (\varepsilon)+0.00265^{n} \sin (\Omega)+0.000063^{\prime \prime} \sin (2 \Omega) .
$$

Where $\varepsilon$ is the mean obliquity of the ecliptic, $\psi$ the luni-solar precession, $\Omega$ the mean longitude of the ascending node of the lunar orbit (18.6 yr period) and $\Delta \psi, \Delta \varepsilon$ are, respectively, the nutation in ecliptic longitude and obliquity.

The complete equation (2) can only be obtained by using implicitly the concept of nonrotating origin, the two last terms of (3) express a correction for the accumulated effect of nutation, on the moving equator, between the epoch of reference and the date.

In the course of implementing the new approach in astrometric VLBI (Capitaine and Gontier 1990), the comparison of the two procedures disclosed the discrepancy due to the terms neglected in the classical procedure. The parameter usually considered for describing the orientation of the Earth, UT1, is affected by the discrepancy as shown by the Figure 1. 


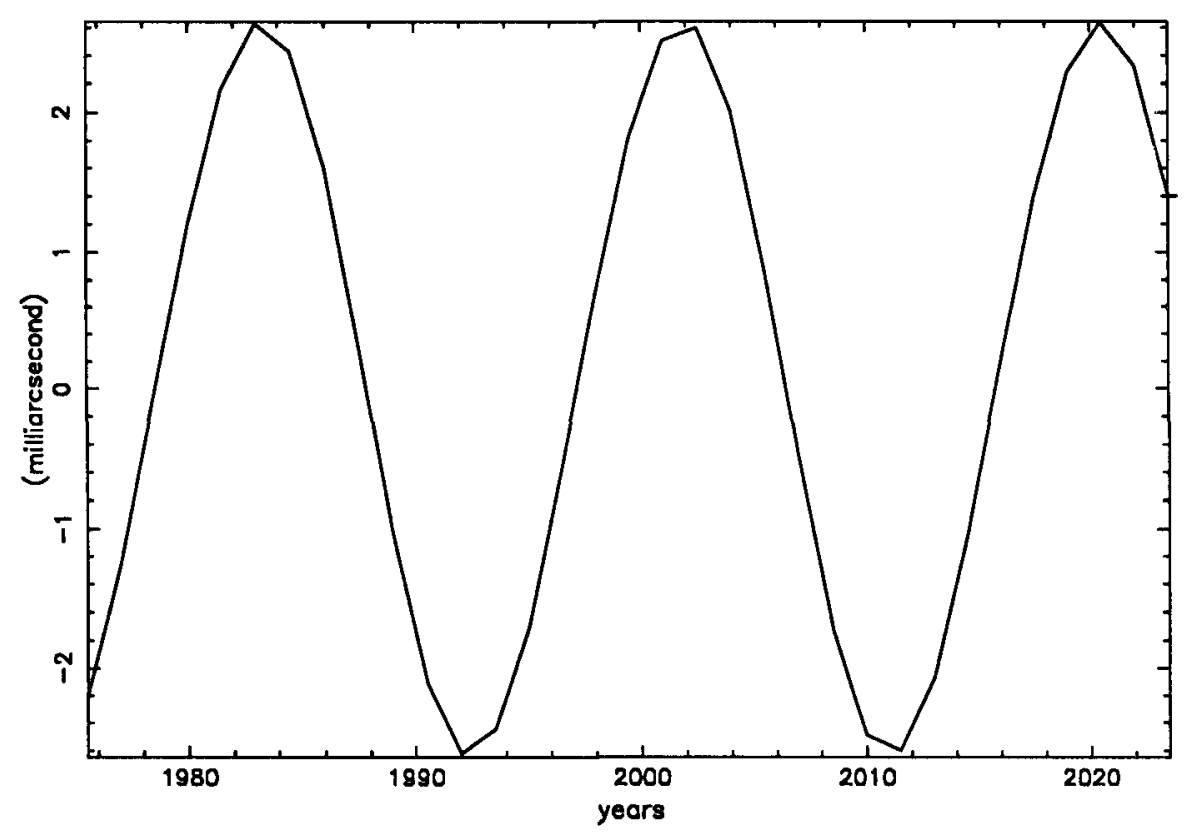

Fig. 1. Differences between the complete procedure and the classical one for the derived UT1.

\section{CONCLUSION}

The introduction in astrometric modelling of observations of the new, realistic, procedure based on the nonrotating origin on the instantaneous equator frees the description of the rotation angle of the Earth (UT1) from the indirect influence of the celestial motions of the pole. This important improvement is illustrated by the disclosure of periodic errors (18.6 years and 9.3 years) at the level of a few milliarcseconds in the expression used in the classical procedure based on the equinox.

The disclosed error in the procedure used up to now is expected to affect mainly the determinations of UT1; however, the existence of small induced effects in the realization of the celestial system should not be ruled out.

\section{REEERENCES}

Aoki, S., Kinoshita, H. 1983, Celest. Mech., 29, p. 335-360.

Capitaine, N. 1990, Celest. Mech .Dyn. Astr., 48, p. 127-143.

Capitaine, N., Guinot, B., Souchay, J. 1986, Celest. Mech., 39, p. 283-307. 
Capitaine, N., Gontier, A.M. 1990, to be published in the proceedings of the colloquium $n 127$ of IAU.

Guinot, B. 1979, in Time and the Earth's Rotation, ed. D.D. McCarthy and J.D. Pilkington, D. Reidel Publ., p. 7-18.

Woolard, E.W. 1953, Astr. Pap. Amer. Naut. Almanach XV, I, p. 1-165. 